\section{Epidemiología de la pancreatitis aguda en Chile entre los años 2013 y 2018}

\author{
ATTILA CSENDES J. ${ }^{1}$, DEYCIES GAETE L. ${ }^{1}$, JAVIER TORO P.. ,a
}

\section{Epidemiology of acute pancreatitis in Chile between 2013 and 2018}

Background: The worldwide incidence of acute pancreatitis $(A P)$ is increasing. Aim: To determine the national incidence of AP between 2013 and 2018. Material and Methods: Analysis of hospital discharge records available at the web page of the Statistical and Information Service of the Chilean Ministry of Health. We recorded the number of patients discharged with a diagnosis of AP, excluding chronic pancreatitis and pancreatic cancer, between 2013 and 2018. We also recorded length of hospital stay, age, etiology, and lethality. Rates of raw and age-adjusted incidence were calculated. Results: During the study period, 46,420 patients with AP were discharged, with an incidence rate ranging between 39 and 43.7/100,000 inhabitants, and a non-significant increase along time of $8.6 \%$. There are important differences between the average adjusted rates of Northern (Arica to Metropolitan) and Southern regions (O'Higgins to Magallanes), with rates of 36.9 and 53.6/100,000 inhabitants respectively $(p<0.01)$. The average hospital stay was 11 days. Two thirds of cases were aged between 20 and 64 years. The case fatality was 4.2\%, with no decrease between 2013 and 2018. The mortality rate was 1.6/100,000 inhabitants. Conclusions: The annual incidence of $A P$ is 42.6/100,000 inhabitants, with geographical differences from North to South, which can be associated with the high frequency of biliary tract disease in aboriginal ethnic groups. The age distribution and length hospital stay were stable over time.

(Rev Med Chile 2021; 149: 961-970)

Key words: Chile; Epidemiologic Studies; Pancreatitis.

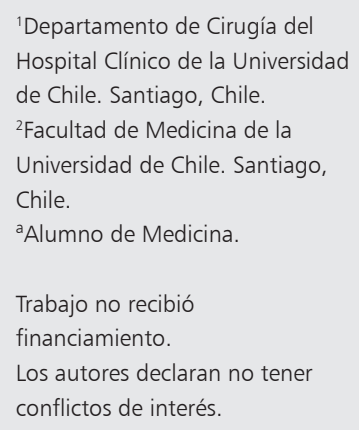

L a pancreatitis aguda (PA) es una patología muy frecuente cuya incidencia mundial ha ido paulatinamente aumentando en el tiempo y varía entre 4,9 y 74,4 x 100.000 habitantes ${ }^{1,2}$. En Estados Unidos de Norteamérica (EE.UU.), durante el 2009 fue la segunda causa de estadía hospitalaria y la quinta en mortalidad por patología digestiva, siendo $30 \%$ más frecuente que el año $2000^{3-5}$. Sin embargo, comparando los años 20022005 con los 2009-2012, la mortalidad disminuyó en 30\%. La incidencia global actual en EE.UU. es de 34 x 100.000 habitantes $^{6-8}$. Existen estadísticas epidemiológicas en varios países europeos con tasas variables, desde 4,6 a 100/100.000 habitantes ${ }^{9}$. En Sudamérica hay escasos datos epidemiológicos al respecto, más bien estadísticas hospitalarias locales, situación que también ocurre en Chile ${ }^{19}$. El estudio más completo corresponde a Berger y cols. ${ }^{11}$, recién publicado, siendo multicéntrico retrospectivo de serie de casos.

El objetivo principal del presente estudio fue determinar la incidencia nacional y regional de la PA entre 2013 y 2018. Como objetivos secundarios, se evaluaron los días de estadía hospitalaria, la distribución por edad, las etiologías de la PA y su letalidad anual. 


\section{Material y Método}

Estudio transversal descriptivo de los egresos hospitalarios por PA entre 2013 y 2018. Las bases de datos se obtienen de la página de Internet del Departamento de Estadísticas e Investigación en Salud (DEIS) y del Instituto Nacional de Estadísticas (https://deis.minsal.cl/ y https://www.ine. $\mathrm{cl} /$ estadisticas/sociales/demografia-y-vitales). Se revisaron los egresos, según edad y región, incluyendo los diagnósticos de PA según los siguientes códigos CIE-10:

- K-850: Pancreatitis idiopática.

- K-851: Pancreatitis biliar.

- K-852: Pancreatitis alcohólica.

- K-853: Pancreatitis por drogas.

- K-858: Pancreatitis por otras causas.

- K-859: Pancreatitis aguda no especificada.

Los diagnósticos de exclusión fueron: pancreatitis crónica y cáncer de páncreas. Se analizaron las siguientes variables:

a. Número total de egresos hospitalarios anual, tasas cruda y ajustada por edad por 100.000 habitantes.

b. Número total de egresos hospitalarios por región y año, considerando las tasas crudas y ajustadas por 100.000 habitantes.

c. Promedio de días de estadía hospitalaria por región y año.

d. Distribución etaria de la PA según los siguientes grupos; < 19 años; 20-44 años; 45-64 años; 65-79 años y $>80$ años.

e. Etiología de la PA, clasificada en: por drogas (PAD), por alcohol (PAA), de etiología idiopática (PAI), y, otras etiologías (PAO), categorías claramente identificadas. El problema se suscitó con la etiología biliar (PAB), que aparece con muy bajo porcentaje y la PA no especifica$\mathrm{da}(\mathrm{PNE})$, que comprende la mayoría. Sabemos que en Chile la patología biliar es responsable de 60 a $82 \%$ de las PA y por eso juntamos la $\mathrm{PAB}$ con la no especificada como PA habitual (PAH), con todos los reparos que esto significa.

f. Porcentaje de letalidad anual y según el grupo etario.

Respecto a la metodología estadística, se utilizó la frecuencia absoluta de los eventos de pancreatitis aguda, calculando la tasa cruda de incidencia por 100.000 habitantes. Para realizar las comparaciones se utilizó la tasa ajustada por edad, estandarizada por método directo con la Población del Censo de 2017 para el total del país y por regiones. Debido a que la división de la Región del Bío Bío se realizó en el 2018, creándose las regiones de Ñuble y del Bío-Bío, se agrupan los eventos de ambas en la última. Para cada media y sus diferencias se calcularon intervalos de confianza del 95\% y se aplicó la prueba de hipótesis de Student, utilizando un valor p significativo menor a 0,05 . Todos los cálculos, tablas y gráficos fueron realizados con el programa Microsoft Excel ${ }^{\circledR} 365$ versión 2019.

\section{Resultados}

En total, hubo 46.420 egresos hospitalarios por PA en los 6 años estudiados, con una tasa de incidencia anual de 39 y 43,7 por 100.000 habitantes en 2013 y 2018 , respectivamente. Al comparar las tasas ajustadas entre ambos años, hay un alza de $8,6 \%$, sin ser estadísticamente significativo (Diferencia $=3,8$ IC95\% $[-2,6-10,2] p=0,249)$. Las tasas anuales se resumen en la Tabla 1.

La tasa de incidencia promedio del país del período entre 2013 y 2018 fue 42,6/100.000 há. La menor incidencia se observó en Atacama entre 2013 y 2014, y, posteriormente, en Arica y Parinacota entre 2015 y 2018. Por otro lado, la Araucanía concentró la mayor tasa del período estudiado. Si excluimos Aysén, con una situación que se explicará luego, aquella con mayor aumento fue Magallanes, con 22,55\% más el 2018 respecto del 2013. La incidencia regional se muestra en la Tabla 2.

Tabla 1. Tasa de incidencia cruda y ajustada por edad según los egresos hospitalarios anuales de pancreatitis aguda entre 2013 y 2018

\begin{tabular}{|cccc|}
\hline Año & Egresos & $\begin{array}{c}\text { Tasa Cruda x } \\
\mathbf{1 0 0 , 0 0 0} \text { há }\end{array}$ & $\begin{array}{c}\text { Tasa Ajustada } \\
\mathbf{x} \mathbf{1 0 0 , 0 0 0} \text { há }\end{array}$ \\
\hline 2013 & 6.873 & 39 & 40,4 \\
\hline 2014 & 7.053 & 39,6 & 40,7 \\
\hline 2015 & 8.018 & 44,6 & 45,5 \\
\hline 2016 & 8.047 & 44,2 & 44,9 \\
\hline 2017 & 8.230 & 44,5 & 44,9 \\
\hline 2018 & 8.199 & 43,7 & 44,2 \\
\hline Total & 46.420 & & \\
\hline
\end{tabular}

Fuente: DEIS. 


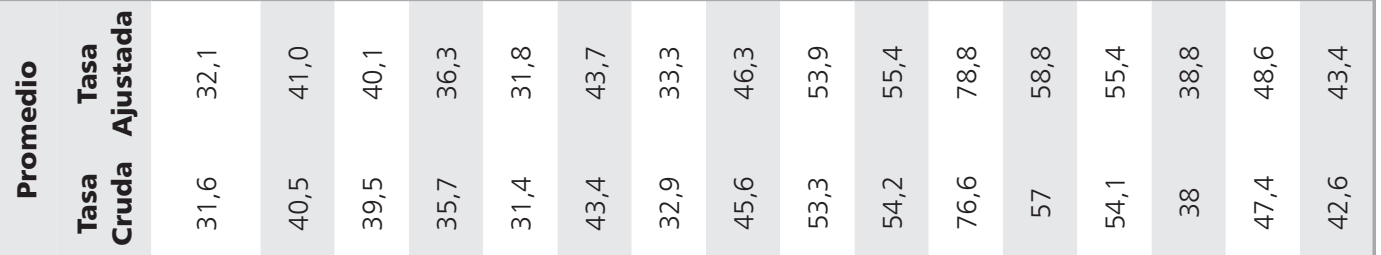

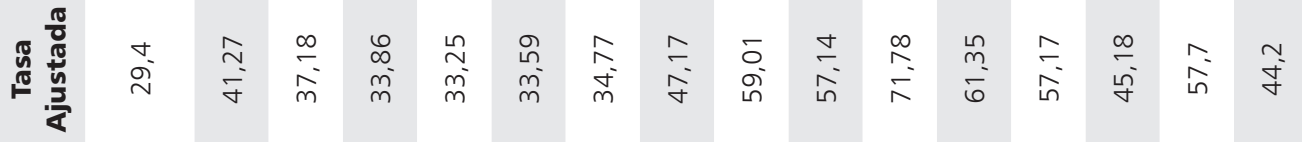

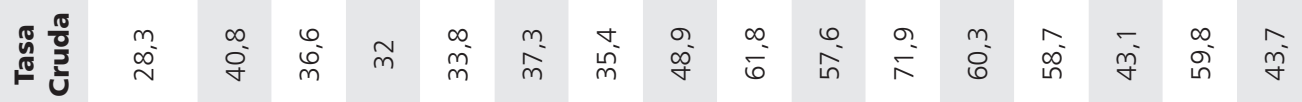

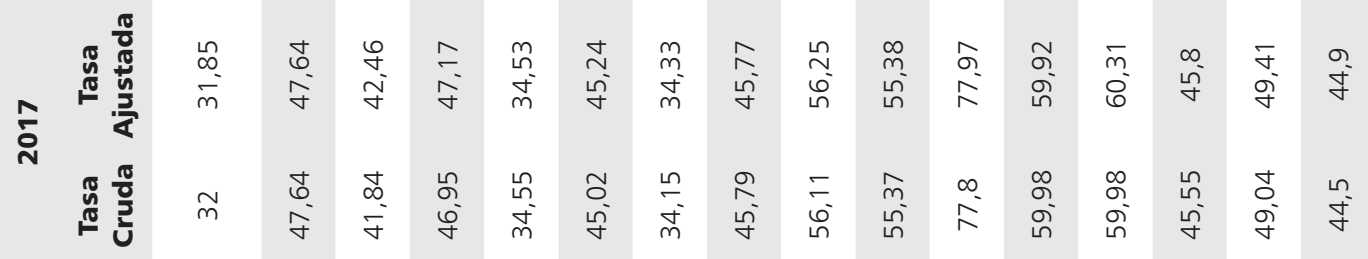

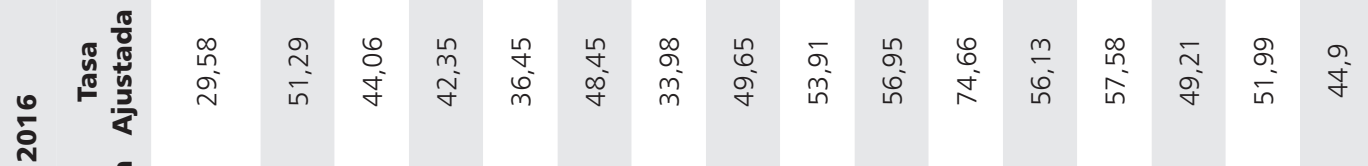

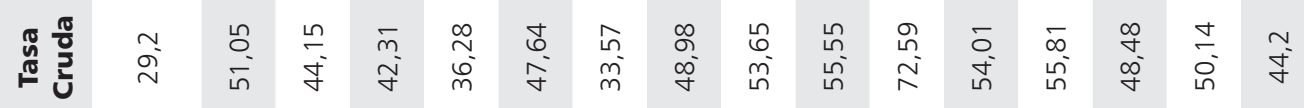

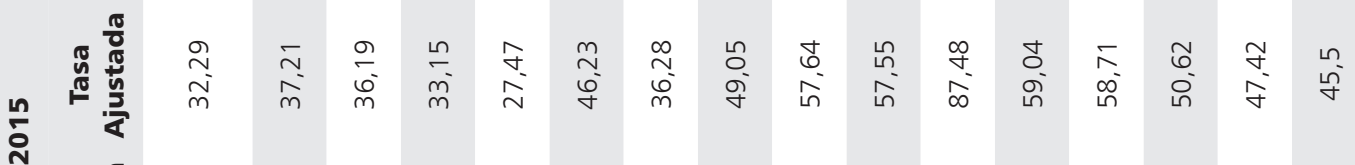

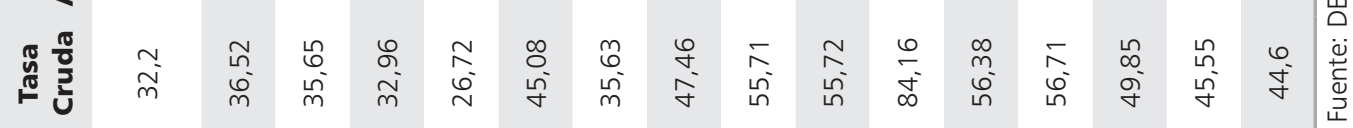

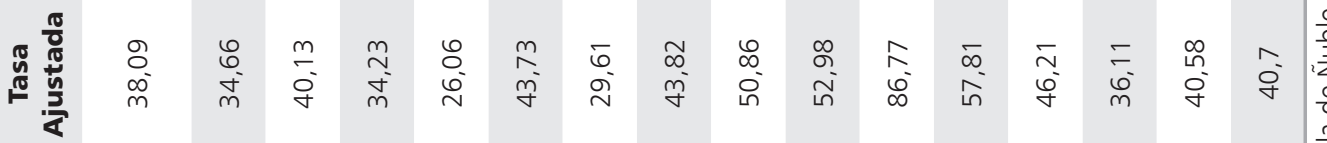

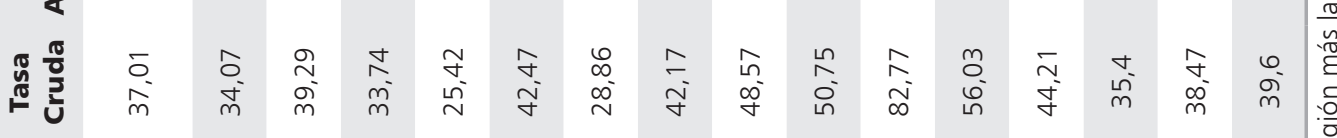

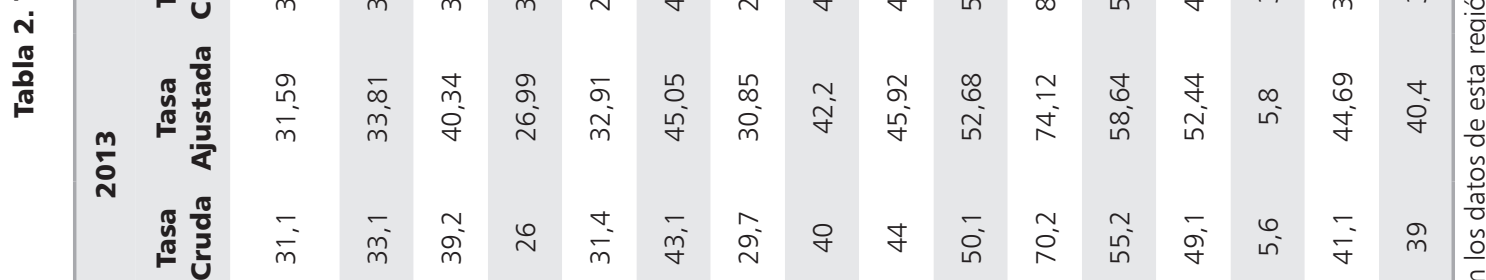

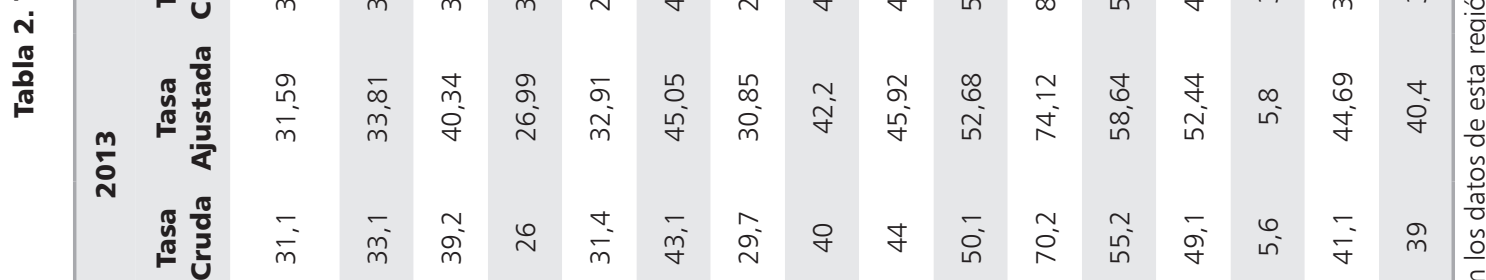

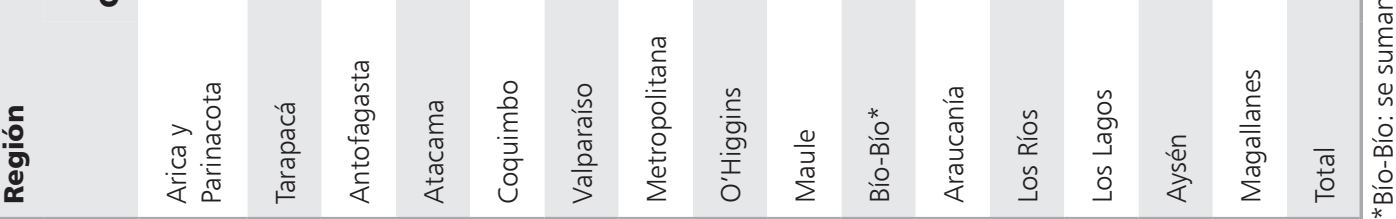


En vista de las diferencias geográficas y epidemiológicas observadas, hemos agrupado las regiones en dos: Grupo 1, desde Arica hasta la Metropolitana, y, Grupo 2, desde O’Higgins hasta Magallanes. En el primero, la tasa de incidencia promedio fue de 36,4/100.000 habitantes y en el segundo de 52,1/100.000 habitantes, con diferencia significativa. Cabe destacar que Aysén se incluye en el Grupo 2 a pesar de que en el año 2013 haya baja incidencia, ya que esto no se condice con la información de los años posteriores, por lo que creemos se debe a un subregistro de casos de PA. En el Grupo 1, las regiones de Arica y Parinacota a la de Valparaíso concentran el 21,85\% de los casos en el año 2013 y el 18,7\% en el 2018, y, la Metropolitana el 31\% y 33\%, respectivamente. Las regiones incluidas en el Grupo 2, acumulan el 47\% de los casos en el 2013 y el 48,6\% en el 2018. Los datos antes mencionados se resumen en la Tabla 3 y se representan en la Figura 1.

Tabla 3. Diferencias entre los Grupos 1 y 2 en las tasa de incidencia de pancreatitis aguda entre los años 2013 y 2018

\begin{tabular}{|cccccccc|}
\hline Año & \multicolumn{2}{c}{ Grupo 1: Arica - Metropolitana } & \multicolumn{2}{c|}{ Grupo 2: O'Higgins - Magallanes } \\
& $\begin{array}{c}\text { Casos } \\
\text { (\% del total) }\end{array}$ & $\begin{array}{c}\text { Tasa } \\
\text { Cruda }\end{array}$ & $\begin{array}{c}\text { Tasa } \\
\text { Ajustada }\end{array}$ & $\begin{array}{c}\text { Casos } \\
\text { (\% del total) }\end{array}$ & $\begin{array}{c}\text { Tasa } \\
\text { Cruda }\end{array}$ & $\begin{array}{c}\text { Tasa } \\
\text { Ajustada }\end{array}$ \\
\hline 2013 & $3.628(52,8 \%)$ & 33,4 & 34,5 & $3.245(47,2 \%)$ & 44,4 & 47,1 \\
\hline 2014 & $3.591(50,9 \%)$ & 34,4 & 35,2 & $3.462(49,1 \%)$ & 49,8 & 51,9 \\
\hline 2015 & $4.160(51,9 \%)$ & 35,0 & 35,5 & $3.862(48,1 \%)$ & 56,4 & 58,4 \\
\hline 2016 & $4.306(53,5 \%)$ & 40,6 & 40,9 & $3.741(46,7 \%)$ & 54,9 & 56,3 \\
\hline 2017 & $4.330(53 \%)$ & 40,3 & 40,5 & $3.900(47 \%)$ & 56,2 & 56,4 \\
\hline 2018 & $4.203(51,3 \%)$ & 34,9 & 34,8 & $3.987(48,7 \%)$ & 57,8 & 57,1 \\
\hline Promedio & & 36,4 & 36,9 & & 52,1 & 53,6 \\
\hline
\end{tabular}

Fuente: DEIS.

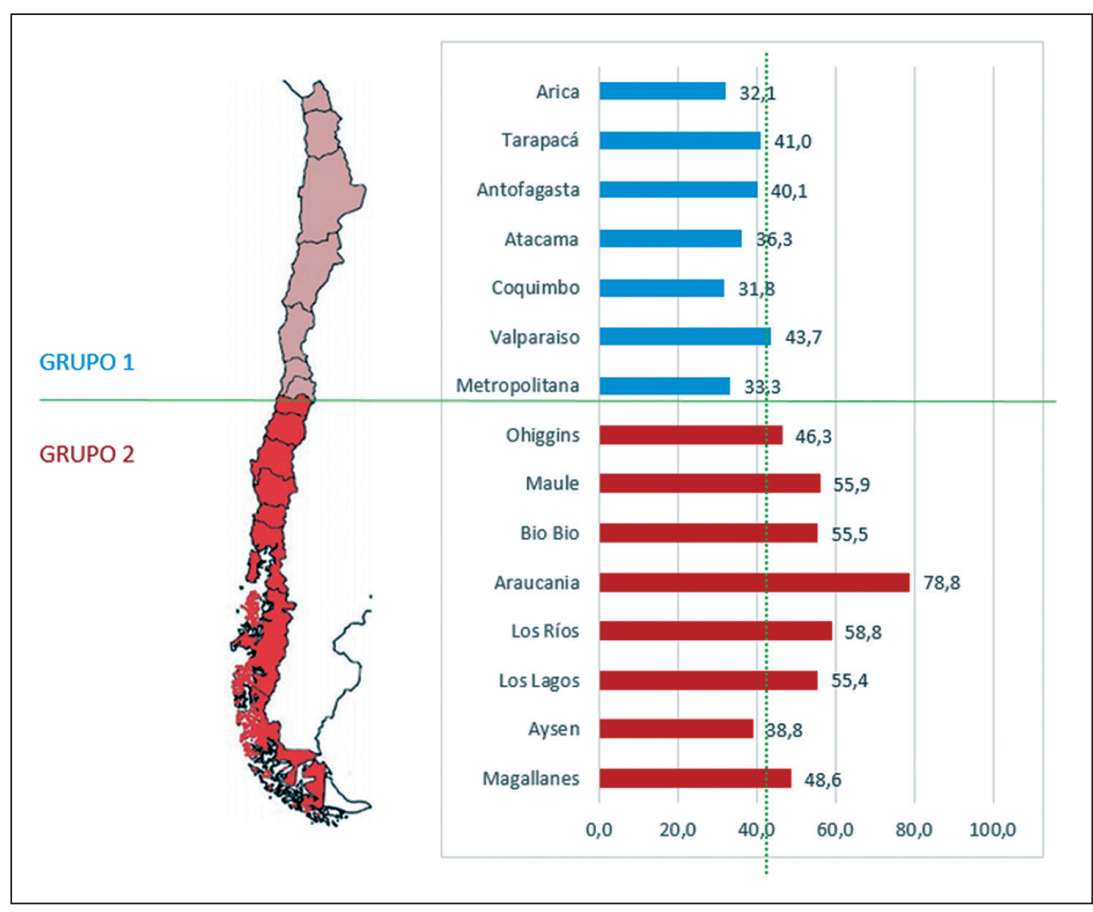

Figura 1. Tasa ajustada promedio de incidencia de pancreatitis aguda en regiones entre 2013 y 2018. Representa el promedio de tasas de incidencia ajustadas de las 15 regiones del país en los seis años, la línea vertical marca la diferencia entre ambos grupos epidemiológicos. Hacia la izquierda están las regiones del Grupo 1 y hacia la derecha, las del grupo 2. 
Las diferencias encontradas entre los grupos son:

a) En el Grupo 1, en cuatro regiones la tasa ajustada promedio fue menor a 40 , en dos fue cercana a 40 y en una región fue de 43,7 . En el Grupo 2, hubo 5 regiones con una tasa superior a 50, dos regiones con tasas de 46 y 48 y solo una región (Aysén) con una tasa de 39.

b) El promedio global de las tasas ajustadas en el total del período estudiado fue de 36,9 para el Grupo 1 y de 53,6 para el Grupo 2, que representa una diferencia de tasas de 16,7 [IC95\% 16,53-16,87], siendo estadísticamente significativa $(\mathrm{p}<0,00)$.

La estadía hospitalaria promedio por PA en el año 2018 fue 11,3 días, con un rango de 6,3 a 19 días. En 13 regiones el promedio fue similar al nacional, y, en dos hay cifras disímiles: Atacama, con 15 días, y Los Ríos, con 8,1. Estos resultados se detallan en la Tabla 4.

La distribución etaria se presenta en la Tabla 5, destacando:

a) Del total de casos de PA, los menores de 19 años representan el 5,1\%, el grupo entre 20 y 44 el 35,1\%; entre los 45 y 64 el 33,6\%; entre 65 y 79 el $18,5 \%$ y, finalmente, el grupo mayor de 80 años el 7,8\%.

b) A pesar de un aumento progresivo de casos a través de los años, la proporción etaria se mantuvo estable durante años.

c) Los grupos de 20-44 y 45-64 años acumularon el 68,7\% de los casos (cerca de 2/3), y los mayores de 65 años acumulan un poco más del $25 \%$ de los casos.
Al comparar las edades por regiones (Tabla 6), encontramos:

a) Tarapacá en el 2013 concentró el 90\% de sus casos entre los 20 y 64 años.

\section{Tabla 4. Estadía hospitalaria promedio y rango de los egresos por pancreatitis aguda del año 2018 según región}

\begin{tabular}{|c|c|c|}
\hline \multirow[t]{2}{*}{ Región } & \multicolumn{2}{|c|}{ Estadía hospitalaria } \\
\hline & $\begin{array}{l}\text { Promedio } \\
\text { Días }\end{array}$ & Rango \\
\hline Arica y Parinacota & 11,6 & $9,3-12,6$ \\
\hline Tarapacá & 11,3 & $10,1-12,1$ \\
\hline Antofagasta & 12,3 & $11,8-13,8$ \\
\hline Atacama & 15 & $11,9-18,3$ \\
\hline Coquimbo & 11,3 & $10-13$ \\
\hline Valparaíso & 11 & $10,5-12,4$ \\
\hline Metropolitana & 11,5 & $10,5-12,4$ \\
\hline O'Higgins & 10,5 & $9,2-11,7$ \\
\hline Maule & 11,2 & $10,1-12,5$ \\
\hline Bío-Bío* & 11 & $10,5-11,9$ \\
\hline Araucanía & 10,5 & $9,5-11,5$ \\
\hline Los Ríos & 8,1 & $6,3-10,3$ \\
\hline Los Lagos & 10,7 & $10-11,5$ \\
\hline Aysén & 10,7 & $10-11,5$ \\
\hline Magallanes & 13,2 & $10,2-19$ \\
\hline Total & 11,33 & $6,3-19$ \\
\hline
\end{tabular}

*En la Región del Bío-Bío se incluyen los datos del Ñuble. Fuente: DEIS.

Tabla 5. Distribución de los casos de pancreatitis aguda entre los años 2013 al 2018 según grupo etario

\begin{tabular}{|ccccccc|}
\hline Año & & \multicolumn{7}{c|}{ Porcentaje del total de casos anuales } & Total casos \\
& $\mathbf{0 - 1 9}$ & $\mathbf{2 0 - 4 4}$ & $\mathbf{4 5 - 6 4}$ & $\mathbf{6 5 - 7 9}$ & $\mathbf{8 0}$ & 6.873 \\
\hline 2013 & 5 & 36,4 & 33,1 & 18,1 & 7,3 & 7.053 \\
\hline 2014 & 5,4 & 34,7 & 34,6 & 18,1 & 7,2 & 8.018 \\
\hline 2015 & 5,5 & 35,2 & 32,9 & 18,7 & 7,9 & 8.047 \\
\hline 2016 & 5,3 & 35,2 & 32,9 & 18,7 & 7,9 & 8.230 \\
\hline 2017 & 4,8 & 34,5 & 34,3 & 18,3 & 8 & 8.199 \\
\hline 2018 & 4,5 & 34,6 & 33,6 & 19 & 8,2 & 100 \\
\hline Promedio $\%$ & 5,1 & 35,1 & 33,6 & 18,5 & 7,8 & 46.420 \\
\hline
\end{tabular}

Fuente: DEIS. 
Tabla 6. Distribución de casos de pancreatitis aguda por grupo etario en regiones en 2013 y 2018

\begin{tabular}{|c|c|c|c|c|c|c|c|c|c|c|}
\hline \multirow[t]{3}{*}{ Región } & \multicolumn{8}{|c|}{ Distribución por edad (\% del total de casos) } & \multicolumn{2}{|c|}{ Total casos } \\
\hline & \multicolumn{2}{|c|}{ 0-19 años } & \multicolumn{2}{|c|}{ 20-44 años } & \multicolumn{2}{|c|}{45 - 64 años } & \multicolumn{2}{|c|}{$>65$ años } & \multirow[b]{2}{*}{2013} & \multirow[b]{2}{*}{2018} \\
\hline & 2013 & 2018 & 2013 & 2018 & 2013 & 2018 & 2013 & 2018 & & \\
\hline Arica y Parinacota & 6,9 & 5,6 & 41,6 & 36,6 & 34,7 & 38 & 16,6 & 19,7 & 72 & 71 \\
\hline Tarapacá & 4,6 & 4,8 & 57 & 41,5 & 32,7 & 37,4 & 5,6 & 16,3 & 107 & 147 \\
\hline Antofagasta & 7,6 & 2,9 & 39,2 & 38,6 & 35 & 39 & 18,1 & 19,3 & 237 & 238 \\
\hline Atacama & 6,3 & 5,7 & 38 & 27,9 & 39,2 & 51,9 & 16,4 & 14,4 & 79 & 104 \\
\hline Coquimbo & 7,6 & 4,4 & 34 & 37,1 & 25,1 & 23,9 & 33,2 & 34,5 & 235 & 272 \\
\hline Valparaíso & 4,8 & 3,3 & 32,4 & 30 & 36,1 & 36,1 & 26,7 & 30,5 & 772 & 701 \\
\hline Metropolitana & 5,6 & 4,5 & 37,4 & 36,3 & 32,9 & 34,2 & 24,1 & 25 & 2.126 & 2.679 \\
\hline O’Higgins & 4,4 & 6,5 & 31,5 & 36,2 & 36 & 28,8 & 28 & 28,4 & 361 & 461 \\
\hline Maule & 6,6 & 3,9 & 36,9 & 32,4 & 31,2 & 31,6 & 25,2 & 32 & 452 & 658 \\
\hline Bío-Bío* & 4,8 & 5,1 & 34,8 & 31,3 & 33,5 & 33,8 & 26,6 & 29,9 & 1.046 & 1.241 \\
\hline Araucanía & 3,6 & 3,6 & 39,2 & 39,3 & 31 & 30,7 & 26 & 26,9 & 686 & 725 \\
\hline Los Ríos & 5,9 & 5,2 & 34 & 32,1 & 25,9 & 29,7 & 34,1 & 32,9 & 220 & 249 \\
\hline Los Lagos & 3,4 & 4,1 & 34,4 & 31,9 & 33,2 & 36,4 & 29 & 27,5 & 407 & 505 \\
\hline Aysén & 0 & 8,3 & 66,7 & 39,6 & 16,7 & 35,4 & 16,7 & 16,7 & 6 & 48 \\
\hline Magallanes & 3 & 6 & 25,4 & 30 & 34,3 & 36 & 37,3 & 28 & 67 & 100 \\
\hline Total (n) & 357 & 369 & 2.488 & 2.834 & 2.263 & 2.758 & 1.765 & 2.238 & 6.873 & 8.199 \\
\hline Total (\%) & 5,2 & 4,5 & 36,2 & 34,5 & 32,9 & 33,6 & 25,7 & 27,4 & 100 & 100 \\
\hline
\end{tabular}

*En la Región del Bío-Bío se incluyen los datos del Ñuble. Fuente: DEIS.

b) Atacama en el 2018 acumuló el 80\% de sus casos entre los 20 y 64 años.

c) Coquimbo presentó la menor incidencia de PA en el grupo de 20 a 64 años en el 2013 y 2018 (59\% y $61 \%)$.

En cuanto a la etiología de la PA, los datos son los más controversiales, ya que no son fidedignos. Al juntar PA biliar con la PA no especificada como $\mathrm{PAH}$, corresponde al $82-83 \%$ del total de casos, la PAA al 1,8\%, la PAD al 0,2\%, la PAI al 6 al 10\% y las otras PA entre el 5 y $8 \%$. La distribución etiológica del 2013 y 2018 por región se encuentra en la Tabla 7.

La letalidad promedio de la PA entre los años 2013 y 2017 (Tabla 8) fue de 4,2\%, con un punto porcentual menor entre 2013 y 2017, no significativo $(p>0,05)$. Los mayores de 80 años presentaron mayor de letalidad, con $14,2 \%$, lo que significa que 1 de cada 7 pacientes fallece, en contraste la letalidad entre 20-44 años, es de 1 por cada 100 pacientes. La Figura 2 grafica la letalidad según grupo etario. La mortalidad específica por PA se encuentra en la Tabla 9, variando entre 1,8 y 1,6 entre 2013 y 2017, sin diferencias significativas.

\section{Discusión}

La incidencia global de la PA citada en artículos previos presenta un amplio grado de estimación, principalmente basado en una mezcla de estudios 
Epidemiología de la Pancreatitis Aguda en Chile - A. Csendes et al

Tabla 7. Distribución de la etiología de las pancreatitis agudas en todas las regiones en los años 2013 y 2018

\begin{tabular}{|c|c|c|c|c|c|c|c|c|c|c|c|c|}
\hline \multirow[t]{3}{*}{ Regiones } & \multicolumn{12}{|c|}{ Etiologías de las Pancreatitis aguda ( $\%$ del total regional) } \\
\hline & \multicolumn{2}{|c|}{ РАH } & \multicolumn{2}{|c|}{ PAA } & \multicolumn{2}{|c|}{ PAD } & \multicolumn{2}{|c|}{ PAI } & \multicolumn{2}{|c|}{ PAO } & \multicolumn{2}{|c|}{ Total casos } \\
\hline & 2013 & 2018 & 2013 & 2018 & 2013 & 2018 & 2013 & 2018 & 2013 & 2018 & 2013 & 2018 \\
\hline Arica y Parinacota & 81,9 & 90 & 0 & 0 & 1,4 & 0 & 0 & 0 & 16,6 & 9,8 & 72 & 71 \\
\hline Tarapacá & 64,5 & 78,9 & 4,7 & 4,1 & 0 & 0 & 13 & 8,2 & 17,8 & 8,8 & 107 & 147 \\
\hline Antofagasta & 58,6 & 72,2 & 0 & 0 & 0 & 0 & 35,9 & 18,5 & 5,5 & 9,2 & 237 & 238 \\
\hline Atacama & 77,2 & 87,5 & 2,5 & 0 & 0 & 0 & 12,6 & 5,8 & 7,6 & 6,7 & 79 & 104 \\
\hline Coquimbo & 45,5 & 78,3 & 0,9 & 0,7 & 0 & 0 & 48,5 & 11,8 & 5,1 & 9,2 & 235 & 272 \\
\hline Valparaíso & 79,5 & 80,1 & 1,7 & 0,8 & 0,5 & 0,3 & 14,8 & 12,1 & 3,5 & 5,8 & 772 & 701 \\
\hline Metropolitana & 82,8 & 80,2 & 1,5 & 2,4 & 1,4 & 5,2 & 10 & 5,4 & 5,9 & 11,4 & 2.126 & 2.679 \\
\hline O'Higgins & 75,3 & 77,4 & 0,9 & 0,7 & 0 & 0,2 & 6,6 & 9,5 & 17,2 & 12,1 & 361 & 461 \\
\hline Maule & 89,8 & 92,8 & 0,2 & 2,4 & 0,2 & 0,2 & 9,7 & 2,4 & 1,6 & 2,1 & 452 & 658 \\
\hline Bío-Bío* & 88,2 & 90,1 & 2,5 & 0,9 & 0,1 & 0,1 & 4,6 & 4,1 & 4,6 & 4,7 & 1.048 & 1.241 \\
\hline Araucanía & 96,5 & 90,5 & 0,7 & 1,9 & 0,2 & 0 & 0,9 & 1,8 & 1,7 & 5,8 & 686 & 724 \\
\hline Los Ríos & 90 & 84,3 & 0,9 & 2,8 & 0 & 0 & 8,6 & 9,6 & 0,5 & 3,2 & 220 & 249 \\
\hline Los Lagos & 83,8 & 80,4 & 5,4 & 3 & 0 & 0,2 & 6,1 & 10,3 & 4,7 & 6,1 & 407 & 505 \\
\hline Aysén & 100 & 95,8 & 0 & 0 & 0 & 0 & 0 & 0 & 0 & 4,2 & 6 & 48 \\
\hline Magallanes & 89,6 & 78 & 0 & 0 & 0 & 0 & 6 & 17 & 4,4 & 5 & 67 & 100 \\
\hline Total (n) & 5.671 & 6.855 & 116 & 145 & 12 & 20 & 712 & 541 & 366 & 638 & 6.877 & 8.199 \\
\hline Total (\%) & 82,4 & 83,6 & 1,7 & 1,8 & 0,2 & 0,2 & 10,3 & 6,6 & 5,3 & 7,8 & 100 & 100 \\
\hline
\end{tabular}

*Bío-Bío: incluye la región del Nuble. PAB+PNE: Pancreatitis aguda biliar y pancreatitis no especificada. PAA: Pancreatitis aguda alcohólica. PAD: Pancreatitis por drogas. PAI: Pancreatitis aguda idiopática. PAO: Pancreatitis aguda otras. Fuente: DEIS.

Tabla 8. Letalidad de la pancreatitis aguda entre los años 2013 y 2017 según rango etario

\begin{tabular}{|c|c|c|c|c|c|c|c|c|}
\hline \multirow[t]{2}{*}{ Año } & \multicolumn{5}{|c|}{ Grupo etario } & \multirow{2}{*}{$\begin{array}{c}\text { Total } \\
\text { Fallecidos }\end{array}$} & \multirow{2}{*}{$\begin{array}{l}\text { Total } \\
\text { casos }\end{array}$} & \multirow{2}{*}{$\begin{array}{c}\text { Tasa de letalidad } \\
\text { total }\end{array}$} \\
\hline & $0-19$ & $20-44$ & $45-64$ & $65-79$ & $\geq 80$ & & & \\
\hline 2013 & 0,8 & 1,3 & 4,1 & 8,3 & 15,9 & 313 & 6.873 & 4,6 \\
\hline 2014 & 0,3 & 1,3 & 3,8 & 9,2 & 11,9 & 306 & 7.053 & 4,3 \\
\hline 2015 & 0,2 & 1 & 3,5 & 7,8 & 15,7 & 340 & 8.018 & 4,2 \\
\hline 2016 & 0,5 & 1,1 & 3,2 & 8 & 15,4 & 337 & 8.049 & 4,2 \\
\hline 2017 & 0,2 & 1 & 4,4 & 7 & 12,3 & 295 & 8.230 & 3,6 \\
\hline Promedio & 0,4 & 1,1 & 3,8 & 8,1 & 14,2 & NA & NA & 4,2 \\
\hline
\end{tabular}

Fuente: DEIS e INE, cuadernos de estadísticas vitales. 


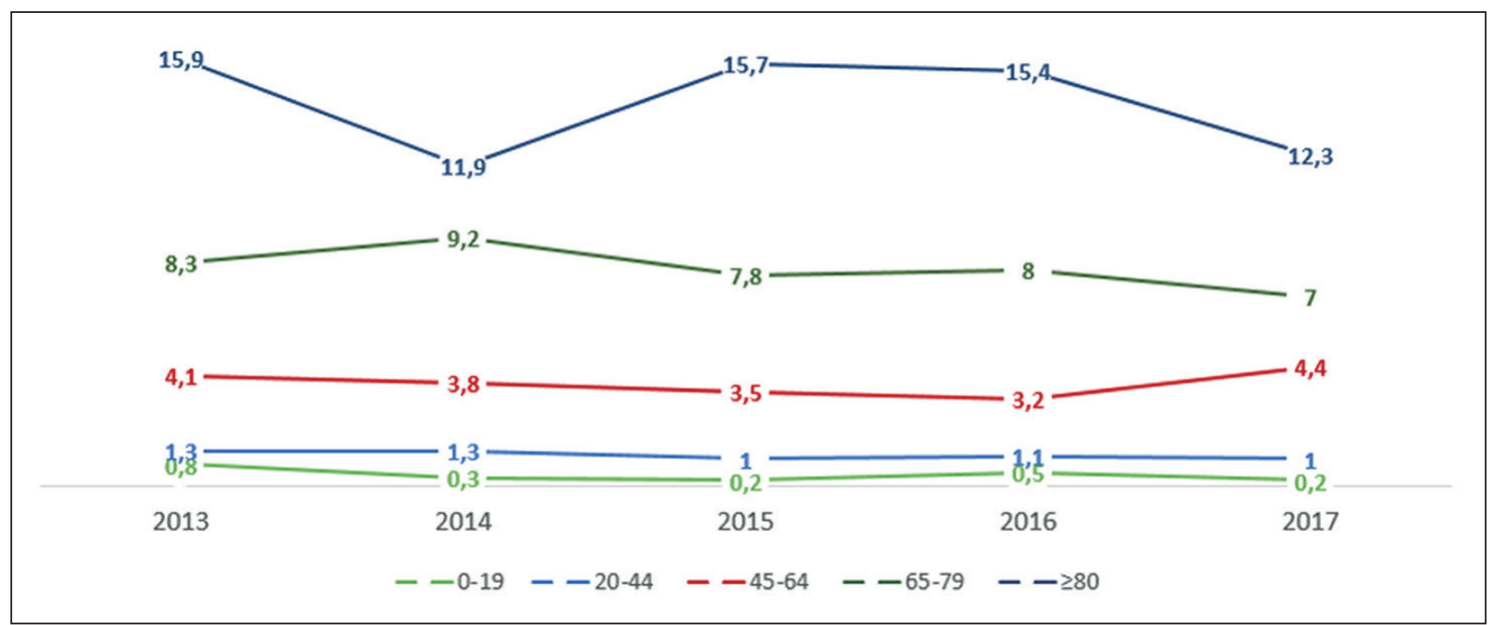

Figura 2. Porcentaje de letalidad de la pancreatitis aguda entre los años 2013 y 2017 según rango etario. Gráfico lineal que representa la letalidad promedio según grupo etario en cada uno de los años estudiados, demostrando que no hay variaciones a lo largo de los 5 años.

Tabla 9. Tasa de mortalidad específica por pancreatitis aguda $\times \mathbf{1 0 0 . 0 0 0}$ habitantes entre el 2013 y 2017

\begin{tabular}{|ccc|}
\hline Año & $\begin{array}{c}\text { Tasa de } \\
\text { mortalidad }\end{array}$ & $\begin{array}{c}\text { Tasa ajustada } \\
\text { de mortalidad }\end{array}$ \\
\hline 2013 & 1,8 & 2 \\
2014 & 1,7 & 1,88 \\
2015 & 1,9 & 2,07 \\
2016 & 1,9 & 1,99 \\
\hline 2017 & 1,6 & 1,62 \\
\hline
\end{tabular}

Fuente: INE, cuadernos de estadísticas vitales.

heterogéneos con diferentes metodologías ${ }^{8}$. Xiao y cols. ${ }^{12}$ determinaron una incidencia global de PA de 34/100.000 habitantes en más de treinta estudios de cohortes poblacionales de alta calidad, sin diferencias por sexo, destacando que no hay datos precisos en Sudamérica.

La tasa de incidencia promedio en 6 años en nuestro país fue $42,6 \times 100.000$ habitantes con tendencia al ascenso. Esta cifra significa que la PA en Chile representa una de las mayores causas de hospitalización por enfermedades gastrointestinales y hepáticas no infecciosas, solo superado probablemente por la colecistolitiasis.

El único dato previo es el de Berger y cols ${ }^{11}$, quienes mencionan una tasa de PA de 40 x 100.000 habitantes, cifra muy similar al nuestra. En el resto de Sudamérica hay pocos datos epidemiológicos ${ }^{13}$. Un estudio en Brasil reporta una tasa de $16 \mathrm{x}$ 100.000 habitantes en el 2006 ${ }^{14}$. En Perú, dos estudios señalan una cifra promedio de $28 \times 100.000$ habitantes ${ }^{15,16}$. En Argentina no hay datos precisos, solo un estudio multicéntrico de 23 hospitales ${ }^{17}$.

Un hallazgo destacable fue el comportamiento epidemiológico de la PA a lo largo de Chile con diferencias significativas entre 7 regiones del "Norte-Centro" comparados con 8 regiones del "Centro-Sur". Este hecho fue señalado por Berger $y$ cols $^{11}$, al notar que en la comuna de Angol (Grupo 2 de nuestro estudio), la tasa de PA era de $57 \mathrm{x}$ 100.000 habitantes. Además, observamos que $7 \mathrm{de}$ las 8 regiones del Grupo 2 tienen mayores tasas que cualquiera del Grupo 1. La diferencia se encontró en los 6 años y, por lo tanto, no es una casualidad, abriendo nuevas interrogantes para tratar de explicarlo. En la Araucanía está la mayor tasa de incidencia promedio en los 6 años con 76,6 x 100.100 habitantes. En esta región y en varias del Grupo 2, hay una importante población mapuche con alta prevalencia de colecistolitiasis ${ }^{18,19}$. Desconocemos si existen diferencias importantes en la dieta del norte y sur de Chile u otros cambios ambientales, que puedan explicar lo anterior, como también de la carga genética que puede llevar a presentar PA entre los diferentes grupos étnicos. 
La distribución etaria fue similar durante los años y en cada una de las regiones. Casi 69\% de las PA ocurrieron entre 20 y 64 años, lo que coincide con el estudio de Berger y cols. ${ }^{11}$ de 962 pacientes con PA con un promedio de 48 años. Destaca que 5\% (2.363) de las PA ocurrió en menores de 19 años, no reportado anteriormente en nuestro país, pues en el estudio mencionado el mínimo de edad fue 19 años. No encontramos datos epidemiológicos en estudios pediátricos en Chile. Por otro lado, no nos referimos a diferencias de género, pero no se describen diferencias en otros reportes ${ }^{8,11}$.

La estadía hospitalaria promedio fue 11 días, siendo en el estudio de Berger y cols. ${ }^{11}$ de 9 , con diferencias según la gravedad: 8 días en PA leve (73\%), 12 en moderada (13,5\%) y 21 días para la grave (13,5\%). Gompertz y cols. ${ }^{10}$ reportan 1.367 pacientes con PA y estadía hospitalaria de 14 días. Mandalia y cols. ${ }^{5}$ señalaron que en los años 2009-2012, la hospitalización disminuyó en 0,78 días, al igual que el costo hospitalario total. Si se considera que el día cama tiene un costo cercano a 1.000 USD, 11 días de hospitalización corresponden a 11.000 USD y en 8.000 pacientes anuales representa un costo promedio de 88 millones de dólares americanos para el país.

La letalidad promedio de la PA fue $4,2 \%$, con un descenso entre 2013 y 2017 de $4,6 \%$ a 3,6\%, representando a cerca de 300 pacientes fallecidos por año. La tasa de mortalidad fue 1,6 por 100.000 habitantes, cifra similar a reportada en 7 estudios de cohortes con 1,16 x 100.000 habitantes $^{8}$. La mortalidad del Berger y cols. ${ }^{11}$ fue $2,5 \%$, mientras que Gompertz y cols. ${ }^{10}$ informaron un 3\% entre los años 2000 y 2010 en el Hospital Clínico de la Universidad de Chile. La mortalidad depende de muchos factores, tal como la gravedad de la PA, ya que la forma leve exhibe mortalidad de $0,3 \%$ y la grave de $14,3 \%{ }^{11}$. También influye la edad, como se demuestra en el presente estudio, con una cifra de $1 \%$ entre los casos de 20 a 44 años y de $14 \%$ sobre 80 años.

La etiología de la PA es el hallazgo más difícil de interpretar. El DEIS parece preciso al considerar algunas etiologías, como por ejemplo las drogas, con una cifra cercana al $0,2 \%$, y la idiopática, cercana al 8,5\%, siendo esta última de 16,5\% en la publicación de Berger y cols ${ }^{11}$. En "otras causas" se refiere a PA metabólicas, post ERCP, postoperatorias, $\mathrm{PA}$ autoinmune, etc. con $6,5 \%$ en promedio en nuestro estudio, mientras que, en el estudio anterior, la mixta y la metabólica corresponden al 12,6\%. Por otro lado, no fue posible precisar la PA biliar, ya que nosotros la agrupamos con la PA no específica denominándolo como PA habitual. La etiología biliar concentraba el 1,8\%, cuando se sabe que en Chile representa el 61\% de las PA (en mujeres 70\%), mientras que la alcohólica un $8,5 \%$ (en hombres 17\%). Papachristou y cols. ${ }^{2}$ mencionan que en Sudamérica la etiología biliar corresponde al $82 \%$ de las causas. Creemos que esta deficiencia se debe a subregistros estadísticos del alta médica enviados al Ministerio de Salud.

Las limitaciones del presente estudio son varias: no se precisa la gravedad de la PA, las complicaciones que motivaron una estadía prolongada, y la etiología está claramente mal identificada. Asimismo, no hay información sobre los procedimientos quirúrgicos o endoscópicos empleados. A pesar de todo, las fortalezas de los datos obtenidos corresponden al gran número de pacientes incluidos, las cifras de estadía hospitalaria y letalidad y a la distribución geográfica.

En conclusión, la PA en Chile es una enfermedad digestiva de alta prevalencia, con una distribución geográfica peculiar con diferencias significativas de incidencias entre regiones del norte y sur. La distribución por edad se mantiene estable en los años estudiados, con una mayor frecuencia entre los 20 y 64 años. La letalidad de la PA ha ido en lento descenso desde 4,6\% a 3,6\%. La estadía hospitalaria promedio en todo el país es de 11 días, cifra que se ha mantenido a través de los años.

Agradecimientos: Los autores agradecen al Dr. Rafael Araos, Jefe de Epidemiología del Ministerio de Salud (MINSAL), por facilitarnos el contacto con el Departamento en Estadísticas e Información de Salud (DEIS).

\section{Referencias}

1. Tenner S, Baillie J, De Witt J, Vege SS. American College of Gastroenterology: Management of acute pancreatitis. Am J Gastoent 2013; 108: 1400-15.

2. Papachristou GI, Machicado JD, Stevens T, Goenka MK, Ferreira M, Gutiérrez SC, et al. Acute pancreatitis patient registry to examine novel therapies in clinical experience (APPRENTICE): an international, multicenter consortium for the study of acute pancreatitis. Ann Gastroenterol 2017; 30: 106-13. 
3. Yadav D, Lowenfels AB. The epidemiology of pancreatitis and pancreatic cancer. Gastroenterology 2013; 144: 1251-61.

4. Lankisch PG, Banks PA. Acute pancreatitis. Lancet 2015; 386: 85-96

5. Mandalia A, Wansteker EJ, Dimagno MJ. Recent adsvances in understanding and managing acute pancreatitis. F1000Res 2019; 7: 959-71.

6. Peery AF, Dellon ES, Lund J, Crockett SD, McGowan $\mathrm{CE}$, Bulsiewicz WJ, et al. Burden of gastgrointestinal disease in the United States:2012 update. Gastroenterology 2012; 143: 1179-87.

7. Lee PJ, Papachristou GI. New insights into acute pancreatitis. Nat Rev Gastroenterol Hepatol 2019; 16: 47996.

8. Petrov MS, Yadav D. Global epidemiology and holistic prevention of pancreatitis. Nat Rev Gastroenterol Hepatol. 2019 Mar; 16: 175-84.

9. Roberts DE, Morrison-Rees S, John A, William JG. The incidence and etiology of acute pancreatitis across $\mathrm{Eu}-$ rope. Pancreatology 2017; 17: 155-65.

10. Gompertz M, Lara I, Fernández L, MItranda JP, Mancilla C, Watkins G, et al. Mortalidad de la Pancreatitis Aguda: experiencia de 20 años en el Hospital clínico de la Universidad de Chile. Rev Med Chile 2013; 141: 562 7.

11. Berger Z, Mancilla C, Tobar E, Morales MP, Baro M, Carrasco M, et al. Acute pancreatitis in Chile: a multicenter study of epidemiology, etiology and clinical outcome. Retrospective análisis of clinical files. Pancreatology 2020; 20: 637-43.

12. Xiao AY, Tan ML, Wu LM, Asrani VM, Windsor JA,
Yadav D, et al. Global incidence and mortality of pancreatic diseases: a systematic review, meta-analysis, and meta-regression of population-based cohort studies. Lancet Gastroenterol Hepatol. 2016; 1: 45-55.

13. Valdivieso MA, Vargas LO, Arana AP. Situación epidemiológica de la Pancreatitis Aguda en Latinoamérica y alcances sobre el diagnóstico. Acta Gastroent. Latinoam. 2016; 46: 100-3.

14. Campos T, Parreira JG, Utiyama E, Rasslan S. Pesquisa nacional sobre condutas na pancreatite aguda. Rev Col Bras Cir. [periódico na Internet] 2008; 35. Disponível em URL: http://www. scielo.br/rcbc.

15. Ministerio de Salud Pública. Perfil epidemiológico de pacientes en consulta externa y hospitalización. MINSA; Lima, Perú 2009.

16. Acevedo A, Taragona J, Malaga G, Barreda L. Identificando la pancreatitis aguda severa. Rev Gastroent Perú 2011; 31: 236-40.

17. Ocampo C, Kohan G, Leiro F, Basso S, Gutiérrez S, Perna L, et al. Diagnóstico y tratamiento de la Pancreatitis Aguda en Argentina. Resultados de un estudio prospectivo en 23 centro. Acta Gastroent Latinoam 2015; 45: 295-302.

18. Csendes A, Korn O, Medina E, Becerra M, Csendes P. Biliary surgery mortality in Chile in 1990. Cooperative study in 17 hospitals. Rev Med Chile 1993; 121: 937-42.

19. Pérez-Ayuso RM, Hernández V, González B, Carvacho C, Navarrete C, Álvarez M, et al. Historia natural de la colelitiasis: Incidencia de colecistectomía en un área urbana y una rural mapuche, en la última década. Rev Med Chile 2002; 130: 723-30. 